\title{
Signal-to-noise considerations in fiber links with periodic or distributed optical amplification
}

\author{
Amnon Yariv* \\ Ortel Corporation, 2015 West Chestnut Street, Alhambra, California 91803-1542
}

Received March 6, 1990; accepted May 25, 1990

\begin{abstract}
The detected signal-to-noise power ratio is evaluated for fiber links with periodic amplification. It is shown that the highest ratio is achieved in the limit of a distributed amplifier $(g=\alpha)$, but that, alternatively, periodic amplification at intervals of $\alpha^{-1}$ entails a penalty of less than $2 \mathrm{~dB}$ compared with the (ideal) distributed case.
\end{abstract}

In recent years there has been a major effort in the field of optical amplification along fiber links. ${ }^{1-6}$ This effort is spurred mostly by the push to avoid the need for conventional electronic repeaters along the fiber. In this Letter the problem of periodic amplification along a fiber link is considered, and it is shown that the ideal limit is reached in a distributed amplifier in which uniform gain is provided the whole length of the fiber such that the signal power remains constant along the link.

Consider a single optical fiber carrying an optical signal with power $S(z)$, where $z$ is the distance measured from the beginning of the fiber. If the fiber attenuation constant is $\alpha\left(m^{-1}\right)$, the signal power varies as $S(z)=S_{0} \exp (-\alpha z)$ so that the detected signal mean-squared current is

$$
\left\langle i_{s}^{2}(z)\right\rangle=\frac{S^{2}(z) e^{2}}{(h \nu)^{2}}=\frac{S_{0}^{2} e^{2}}{(h \nu)^{2}} \exp (-2 \alpha z),
$$

where $e$ is the electronic charge and an optical modulation index of $m=1$ and a unity detector quantum efficiency are assumed. The shot noise at the output of the detector is given by

$$
\left\langle i_{n}{ }^{2}(z)\right\rangle=\frac{2 e^{2} S(z) \Delta \nu}{h \nu}=\frac{2 e^{2} S_{0} \Delta \nu}{h \nu} \exp (-\alpha z),
$$

where $\Delta \nu$ is the detector, and information, bandwidth. The electronic signal-to-noise (SNR) power ratio is given by

$$
\left.\mathrm{SNR}\right|_{\mathrm{at} z}=\frac{\left\langle i_{s}^{2}(z)\right\rangle}{\left\langle i_{n}{ }^{2}(z)\right\rangle}=\frac{S_{0}}{2 h \nu \Delta \nu} \exp (-\alpha z) .
$$

It follows from Eq. (3) that the SNR decreases as $\exp (-\alpha z)$, i.e., a number of decibels equal to that of the fiber loss. In addition to the degradation of the SNR, the attenuated signal may give rise to a situation in which the shot-noise current at the input to the receiver may become comparable with or smaller than the effective input noise current of the receiver, thus causing further degradation in the SNR at the output of the receiver. This latter problem can be combated by a heterodyne receiver or by employing optical amplification. In the following a number of cases for optical amplification are considered, and expressions for the resulting SNR of the detected electronic signal are derived.

Consider an optical in-line amplifier as shown in Fig. 1. The input signal power is $S_{0}$, and it enters the amplifier in a single transverse (usually the fundamental) fiber mode. The amplified output signal is $G S_{0}$, while $F_{0}$ represents the (optical) amplified spontaneous emission (ASE) power at the output that is generated within the amplifier in a band $\Delta \nu$ centered on the optical signal frequency. This noise power is given by $^{7}$

$$
F_{0}=(G-1) \mu h \nu \Delta \nu,
$$

where $\mu=N_{2}-N_{1}\left(g_{2} / g_{1}\right)$ is the inversion factor of the amplifier.

The SNR power ratio at the input to the amplifier is given according to Eqs. (1) and (2) by

$$
\mathrm{SNR}_{\mathrm{in}}=\frac{\left(\frac{S_{0} e}{h \nu}\right)^{2}}{2 e^{2} \frac{S}{h \nu} \Delta \nu}=\frac{S_{0}}{2 h \nu \Delta \nu} .
$$

The detected signal power at the output is

$$
\left\langle i_{s}{ }^{2}\right\rangle_{\text {out }}=\left(\frac{G S_{0} e}{h \nu}\right)^{2},
$$

while the corresponding shot noise is

$$
\left\langle i_{\text {shot }}{ }^{2}\right\rangle_{\text {out }}=\frac{2 e^{2} G S_{0}}{h \nu} \Delta \nu \text {. }
$$

In addition, we need to consider two new sources of output noise current. The first is the current due to beating at the detector between the signal optical frequencies and the ASE frequencies, originating in the amplifier, which are separated by the rf modulation frequency. Such beating produces output noise cur-

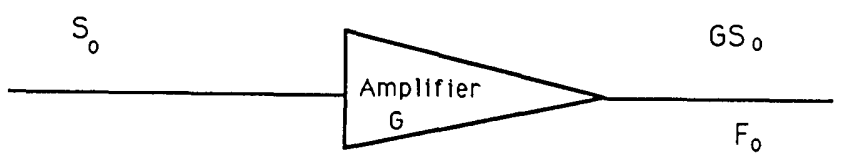

Fig. 1. Optical amplifier with a power gain $G$ and an input signal power $S_{0} . F$ is the total power of the ASE power at the output of the amplifier in the appropriate bandwidth $\Delta \nu$. 
rent components in the same frequency range as that of the signal current. The mean-squared value of this noise current is derivable in a straightforward manner by a method identical to that used to derive the beat current in a heterodyne detector. The result is ${ }^{4}$

$$
\begin{aligned}
\left\langle i_{n}{ }^{2}\right\rangle_{\text {sig-ASE }} & =\frac{4 e^{2} S_{\text {out }} F_{\text {out }}}{(h \nu)^{2}}, \\
& =\frac{4 e^{2} G S_{0}(G-1) \mu h \nu \Delta \nu}{(h \nu)^{2}} .
\end{aligned}
$$

The second noise current is due to the beating of ASE frequencies against themselves. This noise, which is proportional to $F_{0}^{2}$, can be shown to be negligible if the signal power $S(z)$ is not allowed to drop too far. The shot noise due to the ASE has been neglected, which is justified when $S_{0} \gg F_{0}$. The SNR at the output of the amplifier is thus

$$
\mathrm{SNR}_{\text {out }}=\frac{\left(\frac{G S_{0} e}{h \nu}\right)^{2}}{\frac{2 e^{2} G S_{0}}{h \nu} \Delta \nu+\frac{4 e^{2} G(G-1) S_{0} \mu \Delta \nu}{h \nu}},
$$

where a $100 \%$ detector quantum efficiency is assumed. For large gain, $G \gg 1$, the second term in the denominator of Eq. (9) dominates, and

$$
\mathrm{SNR}_{\mathrm{out}} \approx \frac{S_{0}}{4 \mu h \nu \Delta \nu} \text {. }
$$

The ratio of the input SNR to the output SNR is thus

$$
\frac{\mathrm{SNR}_{\text {in }}}{\mathrm{SNR}_{\text {out }}} \approx 2 \mu,
$$

which in an ideal four-level $\left(N_{1}=0, \mu=1\right)$ amplifier is equal to 2 . The single high-gain optical amplifier with pure shot-noise-limited input optical power will thus degrade the SNR of the detected output by a factor with a lower bound (when $\mu=1$ ) of $3 \mathrm{~dB}$. We recall that this degradation is tolerated only in order to save the signal from the far worse fate of succumbing, in its attenuated state, to the receiver noise.

In a very long $(100 \mathrm{~km}<l<5000 \mathrm{~km})$ fiber link we need to amplify the signal a number of times. In what follows, a formalism is developed for systematically treating cascades of amplifiers.

A generalization of Eq. (9) for the SNR of the detected signal at an arbitrary point $z$ along the link is

$$
\operatorname{SNR}(z)=\frac{\left[\frac{e S(z)}{h \nu}\right]^{2}}{\frac{2 e^{2} S(z) \Delta \nu}{h \nu}+\frac{4 e^{2} F(z) S(z)}{(h \nu)^{2}}+\frac{4 k T_{e} \Delta \nu}{R}}
$$

where the last term in the denominator represents the mean-squared thermal noise current of the receiver whose effective noise temperature is $T_{e}$. [ $T_{e}$ is related to the noise figure $F$ of the receiver by $T_{e}=290(F-$ 1).] $R$ is the output impedance of the detector including the receiver's input impedance. Equation (12)

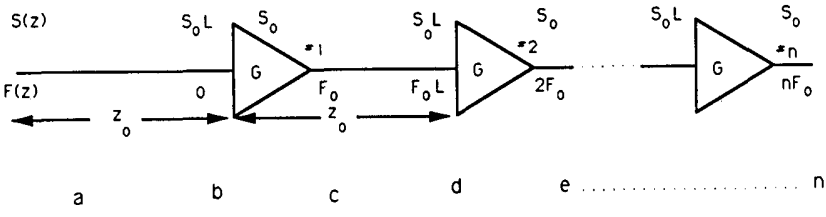

Fig. 2. Fiber link with periodic amplification.

neglects the shot noise due to the ASE, the ASE-ASE beat noise, and the intensity fluctuation noise of the source laser. If $S(z)$ can be maintained by repeated amplification above a certain level, one can neglect the thermal term. [In the numerical example used in connection with Fig. 4, the neglect of the thermal noise is justified for $S(z)>0.1 \mathrm{~mW}$.] Under these realistic circumstances, the SNR Eq. (12) becomes

$$
\operatorname{SNR}(z)=\frac{S^{2}(z)}{2 S(z) h \nu \Delta \nu+4 S(z) F(z)} .
$$

$S(z)$ is the signal power at $z$, while $F(z)$ is the total ASE power at $z$ originating in all the preceding amplifiers $\left(z^{\prime}<z\right)$.

Let us next consider the realistic case of a long fiber with amplifiers employed serially at fixed and equal intervals $\left(z_{0}\right)$ as illustrated by Fig. 2 .

The signal power level $S(z)$ at the fiber input and at the input and output of each amplifier is shown above the axis (the set $S_{0}, S_{0} L, S_{0}, \ldots$ ). The signal is attenuated by a factor of $L \equiv \exp \left(-\alpha z_{0}\right)$ in the distance $z_{0}$ between amplifiers and is boosted back up by the gain $G=L^{-1}=\exp \left(\alpha z_{0}\right)$ at each amplifier. The spontaneous emission power $F(z)$ keeps increasing by increments of $F_{0}$, where $F_{0}$ is given by Eq. (4). Equation (12) is used to calculate the SNR of the detected current at the output of the $n$th amplifier. The result is

$$
\mathrm{SNR}_{n}=\frac{S_{0}}{2 h \nu \Delta \nu\left\{1+2 n \mu\left[\exp \left(\alpha z_{0}\right)-1\right]\right\}},
$$

where, because of the high signal and ASE levels, the thermal receiver noise is neglected. When $G \gg 1$, we find a $z^{-1}$ (more exactly an $n^{-1}$ ) dependence of the SNR rather than the $\exp (-\alpha z)$ behavior predicted by Eq. (3) for a fiber without amplification. The physical reason for this difference is that the repeated amplification keeps the signal level, as well as the level of the signal-ASE beat noise, high. The latter is kept well above the signal shot noise. A fixed amount of beat noise power is thus added at each stage.

Equation (14) suggests that the SNR at $z$ can be improved by reducing $z_{0}$, i.e., using smaller intervals between the amplifiers and reducing the gain $G=$ $\exp \left(\alpha z_{0}\right)$ of each. Although one can take the limit of Eq. (14) as $z_{0} \rightarrow 0$, it is interesting to consider this case $a b$ initio. In this limit a case is reached in which the whole length of the fiber acts as a distributed amplifier with a gain constant $g=\alpha$, just enough to maintain the signal at a constant value. Since $S(z)$ is a constant, we need only evaluate the ASE optical power $F(z)$ in order to obtain an expression for the SNR at $z$. Let us consider a differential length $\mathrm{d} z$. It may be viewed as an amplifier with a gain of $\exp (\mathrm{gdz})$ so that its contribution to $F(z)$ is given by Eq. (4) as 


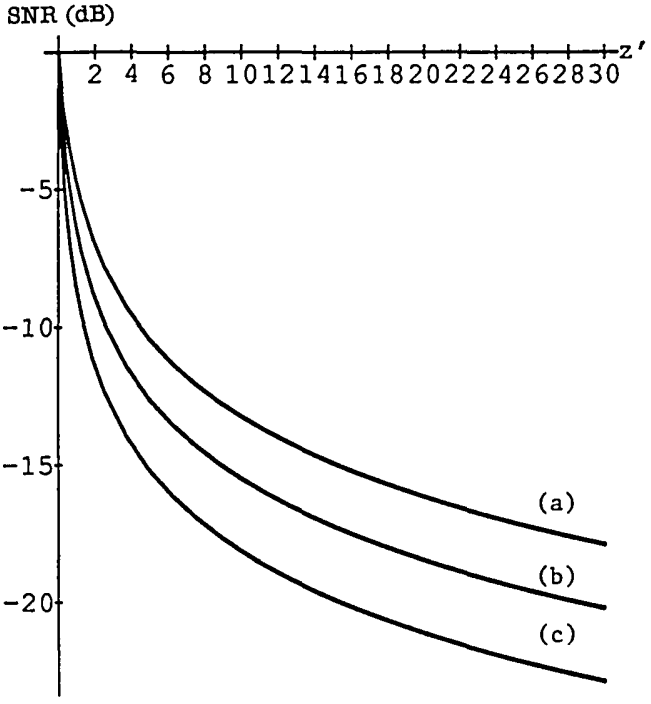

Fig. 3. Universal plot of the degradation of the SNR compared with the initial $(z=0)$ value as a function of $z^{\prime} \equiv \alpha z$ : curve (a), continuous amplification $(g=\alpha)$; curve $(b)$, periodic amplification every $z_{0}=\alpha^{-1}$; curve (c), periodic amplification every $z_{0}=2 \alpha^{-1}$. The horizontal scale is in units of absorption length.

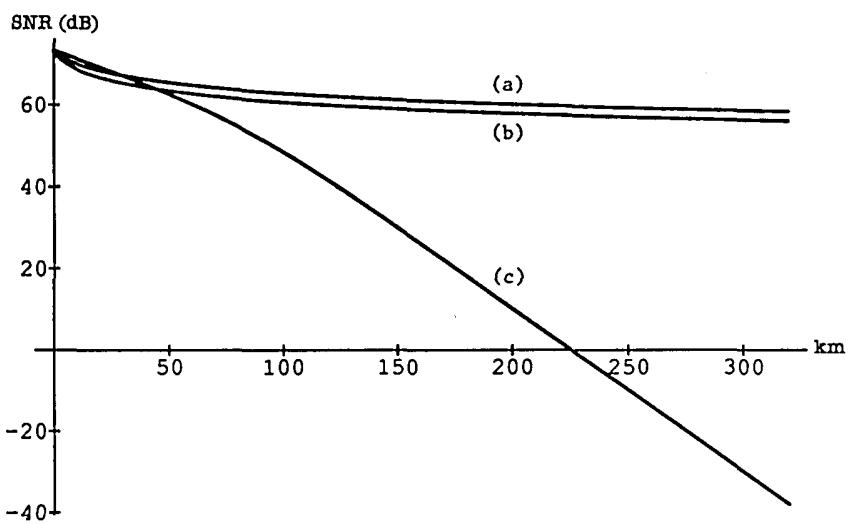

Fig. 4. SNR of detected signal in a fiber link. Curve (a), a continuous amplifier with $g=\alpha, \mu=1$. Curve (b), discrete amplifiers employed every absorption length $\alpha^{-1}=21.76 \mathrm{~km}$ (0.2- $\mathrm{dB} / \mathrm{km}$ fiber loss), $\mu=1$. Curve (c), no amplification and detection with a receiver with a noise figure of $4 \mathrm{~dB}$. The power launched into the fiber is $5 \mathrm{~mW}$, the fiber loss is $0.2 \mathrm{~dB} / \mathrm{km}, \lambda=1.55 \mu \mathrm{m}$, the detection bandwidth is $\Delta \nu=10^{9}$, and the detector load impedance is $1000 \Omega$. Curve (b) is to be read only at multiples of $z^{\prime}=1$, the amplifiers' locations. Curve (c) is to be read at $z^{\prime}=2,4, \ldots$.

$$
\mathrm{d} F=\{\exp [g(\mathrm{~d} z)]-1\} \mu h \nu \Delta \nu
$$

or (recall that $g=\alpha$ )

$$
\frac{\mathrm{d} F}{\mathrm{~d} z}=g \mu h \nu \Delta \nu, \quad F(z)=g \mu h \nu \Delta \nu z,
$$

where $F(0)=0$ is used. By substituting Eq. (16) into Eq. (12) and taking $S(z)=S_{0}$, we have

$$
\operatorname{SNR}(z)=\frac{S_{0}}{2(1+2 \mu \alpha z) h \nu \Delta \nu} \text {. }
$$

As mentioned above, we can also obtain Eq. (17) as the limit of Eq. (14) when $z_{0} \rightarrow 0$. It is interesting to compare the (ideal) distributed amplifier to the discrete amplifier case of Eq. (14),

$$
\operatorname{SNR}(z)=\frac{S_{0}}{2\left\{1+2\left(z / z_{0}\right) \mu\left[\exp \left(\alpha z_{0}\right)-1\right]\right\} h \nu \Delta \nu},
$$

where $G=\exp \left(\alpha z_{0}\right)$ is used.

Figure 3 shows plots of the ideal continuous case described by Eq. (17) as well as two cases of discrete amplifier cascades [Eq. (14)]. The advantage of continuous amplification compared with, say, amplification every $\alpha^{-1}$, is less than $2 \mathrm{~dB}$, so that the latter may be taken as a practical optimum configuration. Figure 4 shows the SNR along a realistic link for continuous amplification [curve (a)], discrete amplifiers spaced by $z_{0}=\alpha^{-1}$ [curve (b)], and no amplification [curve (c)]. The launched power is $P_{0}=5 \mathrm{~mW}, \lambda=$ $1.55 \mu \mathrm{m}, \Delta \nu=10^{9} \mathrm{~Hz}$, and $\alpha=0.2 \mathrm{~dB} / \mathrm{km}$. Curve (b) is to be read only at multiples of $z=\alpha^{-1}=21.7 \mathrm{~km}$, which are the output planes of the optical amplifiers. Curve (c) assumes detection with a receiver with $T_{e}=725 \mathrm{~K}$ $(F=4 \mathrm{~dB})$ and an input impedance of $1000 \Omega$. The advantages of optical amplification for distances exceeding $40 \mathrm{~km}$ in this case are abundantly clear. The break in the slope of curve (c) near $50 \mathrm{~km}$ is due to dominance of receiver noise over signal shot noise from that point on.

There exist other noise mechanisms in fiber systems that were not addressed here. Most of these, such as phase-to-amplitude noise conversion due to Rayleigh scattering in fibers, ${ }^{8,9}$ can be controlled by a variety of means and are deemed nonfundamental.

In conclusion, the problem of cascade amplification has been considered, and it is found that the ideal performance limit is obtained in the case of a uniform distributed fiber amplifier with $g=\alpha$.

* Permanent address, California Institute of Technology, Pasadena, California 91125.

\section{References}

1. J. C. Simon, J. Opt. Commun. 4, 51 (1983).

2. C. R. Giles, E. Desurvire, J. R. Talman, J. R. Simpson, and P. C. Becker, IEEE J. Lightwave Technol. 7, 651 (1989).

3. K. Hagimoto, K. Iwatsuki, A. Takada, M. Nakazawa, M. Saruwatari, K. Aida, K. Nakagawa, and M. Horiguchi, in Digest of Conference on Optical Fiber Communication (Optical Society of America, Washington, D.C., 1989), paper PD15.

4. R. J. Mears, L. Reekie, I. M. Jauncey, and D. N. Payne, Electron. Lett. 23, 1026 (1987).

5. T. Mukai, Y. Yamamoto, and T. Kimura, IEEE J. Quantum Electron. QE-18, 1560 (1982).

6. R. Olshansky, Electron Lett. 24, 1363 (1988).

7. H. Kogelnik and A. Yariv, Proc. IEEE 52, 165 (1964); A. Yariv, Quantum Electronics, 4th ed. (Wiley, New York, 1989), p. 572.

8. A. Judy, presented at the European Conference on Optical Communication, Göteburg, Sweden, 1989, paper TuP-11.

9. A. Yariv, S. Wu, H. Blauvelt, and N. Kwong, in Digest of International Quantum Electronics Conference (Optical Society of America, Washington, D.C., 1990), paper JFA1. 